methods, I still am bold enough to ask whether anything can be "more misleading and inaccurate" than the formula for reduction for temperature and pressure given in both editions of his "Organic Chemistry" under the description of Dumas's vapourdensity method. And to add that no excuse can here be found of a correction given elsewhere, or of the fact that it may be desirable sometimes to state a case broadly to begin with and to define it more closely afterwards. HENRY E. ROSCOE

\section{The Tangent-Galvanometer}

ATTENTION has recently been drawn more than once (notably by Sir William Thomson, and by Mr. W. H. Preece and Mr. Kemp) to the advantages offered, in certain cases of the use of the tangent-galvanometer, by placing the instrument so that the plane of the coils makes a greater or less angle with the plane of the magnetic meridian. It may not be amiss, therefore, to point out that, in $1869, \mathrm{M}$. Bertin showed that the sensitiveness of the tangent-galvanometer for strong currents may be increased and the usable range of deflection doubled by placing the circle in a vertical plane inclined at an angle of $45^{\circ}$ to the magnetic meridian (Annales de Chimie et de Physique, 4th series, vol. xiv. p. 27)

When readings are taken with the current traversing the galvanometer, first in one direction and then in the other, as would always be done if accuracy were important, the expression for the strength of current is almost as simple when the coils make an angle with the magnetic meridian as when they are parallel to it. In the former case the strength of current is

$$
C=\frac{1}{2} k \cos \alpha\left(\tan \theta+\tan \theta^{\prime}\right),
$$

where $\alpha$ is the (fixed) angle between the plane of the coil and the magnetic meridian, and $\theta$ and $\theta^{\prime}$ the deflections of the needle from the plane of the coil due to the current in the two directions. $\theta$ is reckoned positive in the direction from the plane of the coil towards the plane of the meridian, and $\theta^{\prime}$ is reckoned in the opposite direction. To determine the angle $\alpha$ we have

$$
\tan \alpha=\frac{1}{2}\left(\tan \theta-\tan \theta^{\prime}\right),
$$

and it is probably most convenient to determine the numerical value of $\cos a$ in the first expression from this by tables. If the current be adjusted so as to make the second deflection $\theta^{\prime}=0$, we have simply $\tan \alpha=\frac{1}{2} \tan \theta_{1}$, if $\theta_{1}$ be the corresponding deflection with the current reversed.

Universi y College, London, September $30^{\circ}$

\section{Alligators in the Bahamas}

In Catesby's "Natural History of Florida, Carolina, and the Bahama Islands," published in the latter half of the eighteenth century, the author, usually a most accurate observer, states that the mangrove-swamps on the Island of Andros presented a loathsome appearance owing to the remains of fish having been left there, half eaten, by the alligators. During a fairly complete exploration of Andros, which I made in the early part of last year, I observed no traces of these animals, though, as I was not then aware of Catesby's statement, I did not make any special search for them. In response, however, to inquiries made in the local paper, I lately received from the Rev. W. L. de Glanville, Rector of Inagua, Bahamas, a letter from which the following is an extract :-

"Legendary stories of alligators having been floated to this island on logs of mahogany are numerous here. I have not succeeded in verifying any of them. On July $2 \mathrm{r}$, while on a visit to our North-West Point Settlement, a man exhibited to me the skin of an alligator which he had shot a day or two previously. Length all over, from tip to tip, 8 feet; from line at right angles to tip of snout to angle of jaw, I3 inches greatest frontal diameter, about $\mathbf{I}$ inches.

"Logs of mahogany have been drifted recently on these shores, but no one saw an alligator arrive. That shot was on shore, and seen more than once."

As the distance from this to Inagua is more than 400 miles, I have not been able to make inquiries on the spot. But there seems to be no doubt that the alligator must have been carried by the current from the south-east on a log of mahogany or other wood from San Domingo to Inagua. It seems likely that alligators have frequently been drifted to that island, though the absence of a suitable environment has prevented their surviving. The conditions in Andros would suit them better, since about that island there is a considerable extent of fresh and brackish water in lakes, lagoons, and creeks. But it is not easy to understand how they could arrive at Andros, unless we suppose that the Gulf Stream carried them from the north-west coast of Cuba and cast them on the west side of the Great Bahama Bank, whence small local currents and the wind might bring them to the west side of Andros. The distance travelled would in this case be about 300 miles, or about twice as great as that from San Domingo to Inagua.

I trust that this note may be useful as affording further proof of the fact that oceanic currents take some part in the dispersion of even large animals.

Nassau, Bahamas, September 15

\section{Meteors-The September Taurids}

ON September 22, 1886, Ioh. $26 \mathrm{~m}$., I observed a fine meteor absut equal to Jupiter, pursuing a path of some $7^{\circ}$ in the extreme east region of Aries. It left a streak and moved somewhat slowly, being evidently foreshortened near its radiant point in Taurus. At roh. $46 \mathrm{~m}$. the same night I noticed another meteor from the same direction.

On September 21, 1879, I counted 92 meteors, including several from this radiant in Taurus, and on September 22, I884, two others were seen amongst 29 registered on that date.

A comparison of the paths shows a well-defined radiant at $63^{\circ}+23^{\circ}$ (about $8^{\circ}$ N.N.W. of Aldebaran), and I believe the shower is rather an important one, though not well visible until late in the night.

I have recognised several radiants from this position in Taurus in October and November, and in August Mr. Greg derived a shower at $64^{\circ}+22^{\circ}$ from the observations in I 867-74, collected by the Luminous Meteor Committee of the British Association. On October $17-19,1877$, I found a radiant at $63^{\circ}+22^{\circ}$; on November 20,1876 , at $62^{\circ}+22 \frac{1}{2}^{\circ}$; and on November $27, \mathrm{r} 880$, at $63^{\circ}+21^{\circ}$. There are also many other contemporary showers slightly south-west at about $59^{\circ}+20^{\circ}$, and it is in November that the display of meteors from Taurus reaches a maximum.

In September, during the last half of the month, I have determined some other showers in the region of Taurus, supplying meteors of much the same character. The chief additional centres of radiation seem to be at $74^{\circ}+14^{\circ}, 70^{\circ}+4^{\circ}$, and $53^{\circ}+3^{\circ}$, and there is a fairly active shower also from a point further east, at $89^{\circ}+19^{\circ}$, in Orion. The first of these, near II Orionis, was splendidly defined from 8 bright meteors on September 27, I886; and on September 22, I87 I, Lieut.-Col. Tupman saw it at $75^{\circ}+15^{\circ}$. The Orionids at $89^{\circ}+19^{\circ}$ were well seen in September 1877 , and confirmed in September I 884 , but this is essentially a morning shower, as the radiant does not reach a fair altitude for the dispersion of its meteors until the few hours preceding sunrise.

Bristol, October 3

\section{Action of Light upon Diastases}

IN I878, in conjunction with Mr. T. P. Blunt (Proc. Roy. Soc. No. 19I), I showed that the invertive ferment of cane-sugar is destroyed by oxidation on prolonged exposure to surilight. Lately I have extended this observation to other ferments of the like kind, with similar result.

Twenty-five cubic centimetres of very active solutions of malt diastase, pancreatic diastase, and trypsin respectively were rendered inert by insolation in $50 \mathrm{cc}$. flasks for one month (August 25 to September 25 ).

A solution of pepsin was likewise destroyed, but in this case the ferment had been badly prepared, and was not very potent to commence with.

On the other hand, 25 cubic centimetres of solution of rennet, though distinctly enfeebled by insolation, still retained its specific properties at the end of the month. This immunity, however, was only relative, for a more dilute solution in a shallower stratum was almost entirely destroyed by one week's exposure to light (August $3 \mathbf{I}$ to September 7 ).

In all cases the contents of similar flasks kept under like conditions of temperature, \&c., but in the dark, were found to be still active at the termination of the experiment.

In determining the peptonising power of the trypsin and pepsin, Grützner's method (carmine-stained fibrin) was very useful. I find, however, that it is more convenient and econo- 